\title{
Existence Results for an Elliptic Equation of Kirchhoff-Type with Changing Sign Data
}

\author{
By
}

\author{
N. Azzouz and A. BensediK
}

(Université de Tlemcen, Algérie)

\begin{abstract}
Let $\Omega$ be a bounded domain in $\boldsymbol{R}^{N}(N>2)$. We are concerned with the existence and nonexistence of solutions for the following nonlocal problem, $-M\left(\int_{\Omega}|\nabla u(x)|^{2} d x\right) \Delta u=|u|^{p-1} u+\lambda f(x)$ in $\Omega, u_{\left.\right|_{\partial \Omega}}=0$. Where $M$ is continuous function on $\boldsymbol{R}^{+}$and $f \in C^{1}(\bar{\Omega})$ changes sign. $\lambda$ and $p$ are positive parameters. By direct variational method, Galerkin approach and sub and super solutions method some results are established.

Key Words and Phrases. Equation of Kirchhoff-type, Galerkin method, Sub and super solutions, Critical point.

2010 Mathematics Subject Classification Numbers. 35A15, 35A16, 35J25.
\end{abstract}

\section{Introduction}

This paper is concerned with existence and nonexistence of solutions for the problem

$$
\begin{cases}-M\left(\|u\|^{2}\right) \Delta u=|u|^{p-1} u+\lambda f(x) & \text { in } \Omega \\ u=0 & \text { on } \partial \Omega\end{cases}
$$

where $\Omega$ is a bounded domain in $\boldsymbol{R}^{N}(N>2)$ with smooth boundary $\partial \Omega$, $f \in C^{1}(\bar{\Omega})$ changes sign, $M$ is continuous positive function on $\boldsymbol{R}^{+}$and $\lambda$ is a positive parameter.

The problem $(\mathrm{P})$ is the stationary counterpart of the Kirchhoff hyperbolic equation

$$
\frac{\partial^{2} u}{\partial t^{2}}-M\left(\int_{\Omega}|\nabla u(x, t)|^{2} d x\right) \Delta u=g(x, u),
$$

which is motivated in mathematical description of vibrations of an elastic stretched string. The equation $\left(\mathrm{P}^{\prime}\right)$ was proposed by Kirchhoff [6] in 1883 for $M(s)=a s+b ; a, b>0$ and $\Omega=(0, L) \subset \boldsymbol{R}$ as an extension of the classical d'Alembert's wave equation. The model studied takes into account the length changes of the string produced by the transverse vibrations. Such problems are often called nonlocal since the equation is no longer a pointwise identity because 
of the presence of the term $M\left(\|u\|^{2}\right)$. This type of problems received much attention after the work by Lions [7], where a functional analysis framework was proposed. By using variational methods, Alves et al. [2] gave conditions on $M$ and $g$ for which, the stationary problem corresponding to $\left(\mathrm{P}^{\prime}\right)$ possesses positive solutions in the subcritical case. Corrêa and Menezes [4] improved the existence result via Galerkin method when $g(x, u)=g(x)$. They also noticed that the problem admits a positive solution when the function $M$ is bounded and $g(x, u)=\left(u^{+}\right)^{\alpha}+\lambda \varphi(x)$, with $0<\alpha<1$ and $\varphi>0$.

Motivated by the article of Ma [9], the papers [1,2,4] and the work of Dai and $\mathrm{Gu}[5]$ for $M \equiv 1$, we prove that existence and nonexistence of solutions for the problem $(\mathrm{P})$ depend on the positive function $M$, exponent $p$, the parameter $\lambda$ and the function $f$ which is permitted to change sign.

Before the statement of the main results, we make the following hypotheses.

$\left(M_{0}\right) \quad M$ is a continuous function on $\boldsymbol{R}^{+}$such that

$$
M(s) \geq m_{0}, \quad \forall s \geq 0,
$$

for some $m_{0}>0$.

$\left(f_{1}\right) \quad f(x) \in C^{1}(\bar{\Omega})$,

$\left(f_{2}\right) \quad f(x)$ changes sign on $\bar{\Omega}$,

$\left(f_{3}\right)$ the problem

$$
\begin{cases}-\Delta u=f(x) & \text { in } \Omega \\ u=0 & \text { on } \partial \Omega,\end{cases}
$$

has nonnegative solution.

For the existence of such functions $f$, we refer to [3]. In [3], Các et al. treated more general problem. We give here a version of their Theorem 4 for the problem $\left(P_{f}\right)$. Let $G: \Omega \times \Omega \rightarrow \boldsymbol{R}$ be the Green's function for $-\Delta$ with homogenuous boundary condition, and set for $\varepsilon>0$

$$
w_{\varepsilon}(x)=\int_{\Omega} G(x, y)\left[f^{+}(y)-(1+\varepsilon) f^{-}(y)\right] d y,
$$

where $f^{ \pm}(x)=\max ( \pm f(x), 0)$.

Theorem ([3]). Suppose that $\left(f_{1}\right)$ and $\left(f_{2}\right)$ hold. Then if there exists $\varepsilon>0$ such that $w_{\varepsilon}(x)>0$ for all $x \in \Omega$, problem $\left(P_{f}\right)$ has at least one positive solution.

Notation 1. Throughout this paper, we denote by $|\cdot|_{q}$ the $L^{q}$-norm, $1 \leq q \leq \infty$; and by $\|\cdot\|$ the norm of $H_{0}^{1}(\Omega)$ induced by the inner product $(u, v)=\int_{\Omega} \nabla u . \nabla v d x$. The Euclidean norm of $\boldsymbol{R}^{n}$ is denoted by $\|\cdot\|_{n}$ and $\langle.,$. is its inner product. The letter $C$ will denote generic positive constant.

Now, our results are: 
Theorem 1. Assume that $\left(M_{0}\right)$ and $\left(f_{1}\right)$ are satisfied then

(i) if $0<p<1$ problem $(P)$ admits a nontrivial solution for all $\lambda>0$.

(ii) if $1<p<(N+2) /(N-2)$ then there exists $\lambda_{0}>0$ such that problem (P) admits a nontrivial solution for $0 \leq \lambda \leq \lambda_{0}$.

Theorem 2. Assume that $\left(f_{1}\right)$ to $\left(f_{3}\right)$ hold, $p>1$ and $M$ is a nonincreasing function satisfying $\left(M_{0}\right)$. Suppose further that the function $H(t):=t M\left(t^{2}\right)$ is increasing on $\boldsymbol{R}$. Then there exist $0<\lambda_{*}<\lambda^{*}$ such that problem $(P)$ admits at least a positive solution for $\lambda \in\left(0, \lambda_{*}\right)$ and no positive solution for $\lambda>\lambda^{*}$.

Theorem 3. Assume that $\left(f_{1}\right)$ and $\left(f_{2}\right)$ hold, $0<p<1$ and $M$ is a nondecreasing function satisfying $\left(M_{0}\right)$, then problem $(P)$ admits a nontrivial solution with negative energy.

This work is organized as follows. In section 2 we prove Theorem 1 . In section 3 we show the existence of positive solutions when the function $M$ is nonincreasing and we give a nonexistence result. In the last section we treat the case $M$ nondecreasing.

\section{Existence result via Galerkin method}

In this section, we mean by solution of $(P)$ a function $u \in H_{0}^{1}(\Omega)$ such that

$$
M\left(\|u\|^{2}\right) \int_{\Omega} \nabla u . \nabla \varphi d x-\int_{\Omega}\left(|u|^{p-1} u+\lambda f(x)\right) \varphi d x=0 \quad \forall \varphi \in H_{0}^{1}(\Omega) .
$$

The proof of Theorem 2 is based on the following proposition.

Proposition 1 ([8]). Let $F: \boldsymbol{R}^{n} \rightarrow \boldsymbol{R}^{n}$ be a continuous mapping and $B_{r}(O)$ an open ball in $\boldsymbol{R}^{n}$ centered at the origin with radius $r$. If $\langle F(x), x\rangle>0$ for all $x \in \partial B_{r}(O)$, then there exists $x_{0} \in B_{r}(O)$ such that $F\left(x_{0}\right)=0$.

Proof of Theorem 1. Let $\left(e_{k}\right)$ be a complete orthonormal system for $H_{0}^{1}(\Omega)$. For each $n \in N^{*}$ consider the finite dimensional space

$$
V_{n}=\operatorname{span}\left\{e_{1}, \ldots, e_{n}\right\} .
$$

Then $\left(V_{n},\|\cdot\|\right)$ is isometric to $\left(\boldsymbol{R}^{n},\|\cdot\|_{n}\right)$ and for each $v=\sum_{i=1}^{n} \xi_{i} e_{i}$ we have $\|v\|^{2}=\|\xi\|_{n}^{2}=\sum_{i=1}^{n} \xi_{i}^{2}$ where $\xi=\left(\xi_{1}, \ldots, \xi_{n}\right)$. We can make the identification

$$
V_{n} \ni v=\left(\xi_{1}, \ldots, \xi_{n}\right) \in \boldsymbol{R}^{n} .
$$

A function $u_{n} \in V_{n}$ is said to be an approximate solution of the problem $(P)$ if

$$
M\left(\left\|u_{n}\right\|^{2}\right) \int_{\Omega} \nabla u_{n} . \nabla e_{k} d x-\int_{\Omega}\left(\left|u_{n}\right|^{p-1} u_{n}+\lambda f(x)\right) e_{k} d x=0
$$

where $k=1, \ldots, n$. 
Let us define the operator $F: \boldsymbol{R}^{n} \rightarrow \boldsymbol{R}^{n}, F\left(u_{n}\right)=\left(F_{1}\left(u_{n}\right), \ldots, F_{n}\left(u_{n}\right)\right)$ with $F_{k}\left(u_{n}\right)=M\left(\left\|u_{n}\right\|^{2}\right) \int_{\Omega} \nabla u_{n} . \nabla e_{k} d x-\int_{\Omega}\left(\left|u_{n}\right|^{p-1} u_{n}+\lambda f(x)\right) e_{k} d x, \quad k=1, \ldots, n$.

Using the above identification, with $u_{n}=\sum_{i=1}^{n} \xi_{i} e_{i}$, we write

$$
F_{k}\left(u_{n}\right)=M\left(\left\|u_{n}\right\|^{2}\right) \xi_{k}-\int_{\Omega}\left|u_{n}\right|^{p-1} u_{n} e_{k} d x-\lambda \int_{\Omega} f(x) e_{k} d x
$$

and then

$$
\begin{aligned}
\left\langle F\left(u_{n}\right), u_{n}\right\rangle= & M\left(\left\|u_{n}\right\|^{2}\right) \sum_{1}^{n} \xi_{k}^{2}-\int_{\Omega}\left|u_{n}\right|^{p-1} u_{n}\left(\sum_{1}^{n} \xi_{k} e_{k}\right) d x \\
& -\lambda \int_{\Omega} f(x)\left(\sum_{1}^{n} \xi_{k} e_{k}\right) d x \\
= & M\left(\left\|u_{n}\right\|^{2}\right)\left\|u_{n}\right\|^{2}-\int_{\Omega}\left|u_{n}\right|^{p+1} d x-\lambda \int_{\Omega} f(x) u_{n} d x .
\end{aligned}
$$

Using $\left(M_{0}\right),\left(f_{1}\right)$ and Sobolev embedding, we get

$$
\begin{aligned}
\left\langle F\left(u_{n}\right), u_{n}\right\rangle & \geq m_{0}\left\|u_{n}\right\|^{2}-C\left\|u_{n}\right\|^{p+1}-\lambda C|f|_{2}\left\|u_{n}\right\| \\
& \geq\left(m_{0}\left\|u_{n}\right\|-C\left\|u_{n}\right\|^{p}-\lambda C|f|_{2}\right)\left\|u_{n}\right\| .
\end{aligned}
$$

By comparing the curves $C_{1}$ and $C_{2}$ defined by $y=m_{0}^{-1} C t^{p}$ and $y=$ $t-m_{0}^{-1} \lambda C|f|_{2}$ respectively we deduce that

(i) if $0<p<1$ then for large values of $t=\|u\|$, the straight-line $C_{2}$ is above $C_{1}$ for all $\lambda>0$.

(ii) if $1<p<(N+2) /(N-2)$ then for small values of $\|u\|, C_{2}$ is above $C_{1}$ until a limit value $\lambda_{0}>0$.

So in both cases there exists $r=r(\lambda)>0$ such that $\left\langle F\left(u_{n}\right), u_{n}\right\rangle>0$ when $\left\|u_{n}\right\|=r$. Then by Proposition 1, there exists $u_{n} \in V_{n}$, approximate solution of the problem $(P)$ with $\left\|u_{n}\right\|<r$. The obtained sequence $\left(u_{n}\right)$ is bounded so, passing to a subsequence if necessary, there exist $\gamma>0$ and $u \in H_{0}^{1}(\Omega)$ such that

$$
\begin{aligned}
\left\|u_{n}\right\| & \rightarrow \gamma \text { in } \boldsymbol{R} \\
u_{n} & \rightarrow u \text { weakly in } H_{0}^{1}(\Omega) .
\end{aligned}
$$

Fixing $k$ in (1) and letting $n \rightarrow \infty$ we get

$$
M\left(\gamma^{2}\right) \int_{\Omega} \nabla u . \nabla e_{k} d x-\int_{\Omega}\left(|u|^{p-1} u+\lambda f(x)\right) e_{k} d x=0
$$


and since $\left(e_{k}\right)$ is a basis for $H_{0}^{1}(\Omega)$

$$
M\left(\gamma^{2}\right) \int_{\Omega} \nabla u . \nabla \varphi d x-\int_{\Omega}\left(|u|^{p-1} u+\lambda f(x)\right) \varphi d x=0, \quad \forall \varphi \in H_{0}^{1}(\Omega),
$$

in particular for $\varphi=u$ we obtain

$$
M\left(\gamma^{2}\right)\|u\|^{2}-\int_{\Omega}\left(|u|^{p+1}+\lambda f(x) u\right) d x=0 .
$$

Similarly we have from (1)

$$
M\left(\left\|u_{n}\right\|^{2}\right) \int_{\Omega} \nabla u_{n} . \nabla \varphi d x-\int_{\Omega}\left(\left|u_{n}\right|^{p-1} u_{n}+\lambda f(x)\right) \varphi d x=0, \quad \forall \varphi \in V_{n}
$$

for $\varphi=u_{n}$ we obtain

$$
M\left(\left\|u_{n}\right\|^{2}\right)\left\|u_{n}\right\|^{2}-\int_{\Omega}\left(\left|u_{n}\right|^{p+1}+\lambda f(x) u_{n}\right) d x=0
$$

letting $n \rightarrow \infty$ and using the compact embedding $H_{0}^{1}(\Omega) \subset L^{p+1}(\Omega)$ we obtain

$$
M\left(\gamma^{2}\right) \gamma^{2}-\int_{\Omega}\left(|u|^{p+1}+\lambda f(x) u\right) d x=0
$$

By (2) and (3) we get $\gamma=\|u\|$ and so $u_{n} \rightarrow u$ in $H_{0}^{1}(\Omega)$.

Remark 1. We see, from the proof that it is sufficient to take $f \in H^{-1}(\Omega)$ instead of $\left(f_{1}\right)$, where $H^{-1}(\Omega)$ is the dual space of $H_{0}^{1}(\Omega)$.

\section{Proof of Theorem 2}

In this section we suppose that $p>1$ and $M$ is a nonincreasing function satisfying $\left(M_{0}\right)$. We will use the sub and super solutions method. Let $u_{f}$ be a nonnegative solution of problem

$$
\begin{cases}-\Delta u=f(x) & \text { in } \Omega \\ u=0 & \text { on } \partial \Omega\end{cases}
$$

the existence of the solution $u_{f}$ follows from $\left(f_{3}\right)$. We determine a subsolution of $(P)$ in the form

$$
\underline{u}=\gamma u_{f}, \quad \text { with } \gamma \in \boldsymbol{R}^{+}
$$


Then we have

$$
\begin{aligned}
-M\left(\|\underline{u}\|^{2}\right) \Delta \underline{u} & =-M\left(\gamma^{2}\left\|u_{f}\right\|^{2}\right) \gamma \Delta u_{f} \\
& =M\left(\gamma^{2}\left\|u_{f}\right\|^{2}\right) \gamma f(x) .
\end{aligned}
$$

$\underline{u}$ is subsolution of $(P)$ if

$$
-M\left(\|\underline{u}\|^{2}\right) \Delta \underline{u} \leq|\underline{u}|^{p-1} \underline{u}+\lambda f(x) .
$$

To obtain the last inequality we must take $\gamma$ such that

$$
M\left(\gamma^{2}\left\|u_{f}\right\|^{2}\right) \frac{\gamma}{\lambda}=1
$$

because the function $f$ changes sign. Let us prove that the equation (4) admits at least a positive solution $\gamma$.

Put $s=\gamma^{2}\left\|u_{f}\right\|^{2}$, then (4) becomes

$$
\psi(s):=\frac{M(s) \sqrt{s}}{\left\|u_{f}\right\|}=\lambda
$$

We have $\psi(0)=0$ and $\lim _{s \rightarrow+\infty} \psi(s)=+\infty$, and since $\psi$ is continuous on $\boldsymbol{R}^{+}$ there exists $s(\lambda)>0$ such that $\psi(s)=\lambda$. Then there exists $\gamma>0$ satisfying (4), and existence of $\underline{u}$ follows.

Now let us find a supersolution $\bar{u}$ of $(P)$ with the condition $\bar{u}(x) \geq \underline{u}(x)$, $x \in \Omega$. Let $e$ be the unique positive solution of the linear Dirichlet problem

$$
\begin{cases}-\Delta u=1 & \text { in } \Omega \\ u=0 & \text { on } \partial \Omega\end{cases}
$$

Set

$$
\bar{u}=A e, \quad \text { with } A \in \boldsymbol{R}^{+} \text {. }
$$

Then by $\left(M_{0}\right)$

$$
-M\left(\|\bar{u}\|^{2}\right) \Delta \bar{u}=M\left(A^{2}\|e\|^{2}\right) A \geq m_{0} A .
$$

If the positive number $A$ satisfies the following relation

$$
m_{0} A \geq A^{p}|e|_{\infty}^{p}+\lambda|f|_{\infty},
$$

then $\bar{u}$ furnishes a supersolution of $(P)$. To prove the existence of such $A$, consider the function

$$
h(A)=\left(m_{0} A-A^{p}|e|_{\infty}^{p}\right)|f|_{\infty}^{-1} .
$$


Easy computations show that $h$ is concave and achieves its maximum at

$$
A_{0}=\left(\frac{m_{0}}{p|e|_{\infty}^{p}}\right)^{1 /(p-1)} .
$$

Therefore if $0<\lambda<\lambda_{0}=h\left(A_{0}\right)$ then $\bar{u}=A_{0} e$ is a supersolution of the problem $(P)$ because

$$
-M\left(\|\bar{u}\|^{2}\right) \Delta \bar{u} \geq m_{0} A_{0} \geq|\bar{u}|_{\infty}^{p}+\lambda|f|_{\infty} \geq|\bar{u}|^{p-1} \bar{u}+\lambda f(x) .
$$

Now an adequate choice of $0<\lambda<\lambda_{*} \leq \lambda_{0}$ and then of $\gamma$ ensures that

$$
\bar{u}(x) \geq \underline{u}(x), \quad x \in \Omega .
$$

To complete the proof of the existence result we need the following lemma.

Lemma 1 ([1]). Under the hypotheses of Theorem 2, if $v$ and $w$ are nonnegative functions verifying

$$
\begin{cases}-M\left(\|w\|^{2}\right) \Delta w \geq-M\left(\|v\|^{2}\right) \Delta v & \text { in } \Omega \\ v=w=0 & \text { on } \partial \Omega\end{cases}
$$

then

$$
w \geq v \quad \text { in } \Omega \text {. }
$$

Proof. We follow along the lines of [1]. Mutiltiplying both sides of the inequality by $v$ and $w$ and integrating, we get

$$
\frac{M\left(\|w\|^{2}\right)\|w\|^{2}}{M\left(\|v\|^{2}\right)} \geq(w, v) \geq \frac{M\left(\|v\|^{2}\right)\|v\|^{2}}{M\left(\|w\|^{2}\right)}
$$

and so

$$
M\left(\|w\|^{2}\right)\|w\| \geq M\left(\|v\|^{2}\right)\|v\|
$$

i.e.

$$
H(\|w\|) \geq H(\|v\|)
$$

Since $H$ is increasing we obtain $\|w\| \geq\|v\|$ and then

$$
M\left(\|w\|^{2}\right) \leq M\left(\|v\|^{2}\right)
$$

because $M$ is nonincreasing. On the other hand, by application of the maximum principle to $(P 1)$ we get

$$
M\left(\|w\|^{2}\right) w \geq M\left(\|v\|^{2}\right) v
$$

This with (5) yield $w \geq v$. This ends the proof of Lemma 1 . 
Remark 2. Here is an example of function $M$ satisfying the assumptions of Theorem 2. We take

$$
M: \boldsymbol{R}^{+} \rightarrow \boldsymbol{R}^{+}, \quad M(t)=\frac{a t+b}{\alpha t+\beta}+c,
$$

where $a, b, \alpha, \beta$ and $c$ are positive numbers such that

$$
a \beta-b \alpha<0 \quad \text { and } \quad \frac{a+\alpha c}{b+\beta c}>\frac{1}{4} .
$$

Now to obtain a solution of problem $(P)$ we define a sequence $\left(u_{n}\right)_{n \geq 0} \subset$ $H_{0}^{1}(\Omega)$ by $u_{0}=\bar{u}$ and $u_{n}(n \geq 1)$ is the unique solution of the problem

$$
\begin{cases}-M\left(\left\|u_{n}\right\|^{2}\right) \Delta u_{n}=g\left(x, u_{n-1}\right) & \text { in } \Omega \\ u_{n}=0 & \text { on } \partial \Omega,\end{cases}
$$

where, for the sake of simplicity $g(x, t)=|t|^{p-1} t+\lambda f(x)$. The problem (P2) is $M$-linear in the sense that, if $u_{n-1} \in H_{0}^{1}(\Omega)$ is given, the right hand side is independent of $u_{n}$. Then since $H(\boldsymbol{R})=\boldsymbol{R}$ and $g\left(., u_{n-1}\right) \in L^{2}(\Omega)$, we deduce from a result in [1] that problem (P2) admits a unique solution $u_{n} \in H_{0}^{1}(\Omega)$. Now using the fact that $u_{0}$ is a super solution, we write

$$
-M\left(\left\|u_{0}\right\|^{2}\right) \Delta u_{0} \geq g\left(x, u_{0}\right)=-M\left(\left\|u_{1}\right\|^{2}\right) \Delta u_{1},
$$

and by Lemma $1, u_{0} \geq u_{1}$. Also, since $u_{0} \geq \underline{u} \geq 0$ one has

$$
-M\left(\left\|u_{1}\right\|^{2}\right) \Delta u_{1}=g\left(x, u_{0}\right) \geq g(x, \underline{u}) \geq-M\left(\|\underline{u}\|^{2}\right) \Delta \underline{u}
$$

from which, according to Lemma $1, u_{1} \geq \underline{u}$. For $u_{2}$ we write

$$
-M\left(\left\|u_{1}\right\|^{2}\right) \Delta u_{1}=g\left(x, u_{0}\right) \geq g\left(x, u_{1}\right)=-M\left(\left\|u_{2}\right\|^{2}\right) \Delta u_{2},
$$

and then $u_{1} \geq u_{2}$. Similarly $u_{2} \geq \underline{u}$ because

$$
-M\left(\left\|u_{2}\right\|^{2}\right) \Delta u_{2}=g\left(x, u_{1}\right) \geq g(x, \underline{u}) \geq-M\left(\|\underline{u}\|^{2}\right) \Delta \underline{u} .
$$

Repeating this argument we get a bounded monotone sequence $\left(u_{n}\right) \subset H_{0}^{1}(\Omega)$,

$$
\bar{u}=u_{0} \geq u_{1} \geq u_{2} \geq \cdots \geq u_{n} \geq \cdots \geq \underline{u} \geq 0, \quad \underline{u} \neq 0 .
$$

Using bootstrap arguments we show that $\left(u_{n}\right) \subset C^{0, \alpha}(\bar{\Omega}), 0<\alpha<1$, and since $f \in C^{0, \alpha}(\bar{\Omega})$ we have $g\left(., u_{n}\right) \in C^{0, \alpha}(\bar{\Omega})$. The Schauder estimate gives $\left(u_{n}\right) \subset$ $C^{2, \alpha}(\bar{\Omega})$.

Now multiplying (P2) by $u_{n}$, integrating and using Hölder inequality and Sobolev embedding we get

$$
H\left(\left\|u_{n}\right\|\right) \leq C
$$


Using the fact that $H(t) \rightarrow+\infty$ as $t \rightarrow+\infty$, we infer that $\left(u_{n}\right)$ is bounded in $H_{0}^{1}(\Omega)$ and consequently in $C^{2, \alpha}(\bar{\Omega})$ which is compactly embedded in $C^{2}(\bar{\Omega})$. Then $\left(u_{n}\right)$ has a subsequence which converges in $C^{2}(\bar{\Omega})$ to a limit $u \geq \underline{u} \geq 0$. Being monotone, $\left(u_{n}\right)$ converges itself to $u$. Now letting $n \rightarrow \infty$ in (P2), we deduce that $u$ is a positive solution of the problem $(P)$. This completes the proof of existence result for $0<\lambda<\lambda_{*}$.

To prove the nonexistence result of Theorem 2 , let $\lambda_{1}$ be the first eigenvalue of $\left(-\Delta, H_{0}^{1}(\Omega)\right)$ and $\phi_{1}$ the corresponding eigenfunction. If $u_{\lambda}$ is a positive solution of the problem $(P)$ then

$$
-M\left(\left\|u_{\lambda}\right\|^{2}\right) \int_{\Omega} \phi_{1} \Delta u_{\lambda} d x=\int_{\Omega} u_{\lambda}^{p} \phi_{1} d x+\lambda \int_{\Omega} f(x) \phi_{1} d x .
$$

Thus

$$
\lambda \int_{\Omega} f(x) \phi_{1} d x=\int_{\Omega}\left[\lambda_{1} M\left(\left\|u_{\lambda}\right\|^{2}\right) u_{\lambda}-u_{\lambda}^{p}\right] \phi_{1} d x .
$$

The function $M$ is nonincreasing, then

$$
\lambda \int_{\Omega} f(x) \phi_{1} d x \leq \int_{\Omega}\left[\lambda_{1} M(0) u_{\lambda}-u_{\lambda}^{p}\right] \phi_{1} d x .
$$

The maximum of the function $u_{\lambda} \mapsto \lambda_{1} M(0) u_{\lambda}-u_{\lambda}^{p}$ is achieved at $u_{\lambda}=$ $\left(\lambda_{1} M(0) / p\right)^{1 /(p-1)}$. Consequently

$$
\lambda \int_{\Omega} f(x) \phi_{1} d x \leq\left(\frac{\lambda_{1} M(0)}{p}\right)^{p /(p-1)}(p-1) \int_{\Omega} \phi_{1} d x .
$$

Recall that, by condition $\left(f_{3}\right)$ the problem

$$
\begin{cases}-\Delta u=f(x) & \text { in } \Omega \\ u=0 & \text { on } \partial \Omega,\end{cases}
$$

has nonnegative solution $u_{f} \in H_{0}^{1}(\Omega)$ and then

$$
\int_{\Omega} f(x) \phi_{1} d x=\int_{\Omega}-\Delta u_{f} \phi_{1} d x=\lambda_{1} \int_{\Omega} u_{f} \phi_{1} d x>0 .
$$

From this and (6) we get

$$
\lambda \leq \frac{\left(\frac{\lambda_{1} M(0)}{p}\right)^{p /(p-1)}(p-1) \int_{\Omega} \phi_{1} d x}{\int_{\Omega} f(x) \phi_{1} d x}=\lambda^{*} .
$$

This implies that the problem $(P)$ has no positive solution for $\lambda>\lambda^{*}$. 


\section{Proof of Theorem 3}

In this section we suppose that the function $M$ is increasing, satisfies $\left(M_{0}\right)$ and $0<p<1$. We look for solutions which are critical points of the energy functional $I$, defined on $H_{0}^{1}(\Omega)$ by

$$
I(u)=\frac{1}{2} \tilde{M}\left(\|u\|^{2}\right)-\frac{1}{p+1} \int_{\Omega}|u|^{p+1} d x-\lambda \int_{\Omega} f(x) u d x
$$

where $\tilde{M}(t)=\int_{0}^{t} M(s) d s$.

The condition $\left(M_{0}\right)$, Hölder inequality and Sobolev embedding give

$$
I(u) \geq \frac{1}{2} m_{0}\|u\|^{2}-\frac{C}{p+1}\|u\|^{p+1}-\lambda C\|u\||f|_{2} .
$$

Since $1<p+1<2$, the functional $I$ is coercive. Let $\alpha=\inf _{u \in H_{0}^{1}(\Omega)} I(u)$ and $\left(u_{n}\right)$ be a minimizing sequence that is, satisfying $I\left(u_{n}\right) \rightarrow \alpha$. By coerciveness $\left(u_{n}\right)$ is bounded in $H_{0}^{1}(\Omega)$, and so we may assume that $u_{n} \rightarrow v$ weakly for some $v \in H_{0}^{1}(\Omega)$. The function $M$ is positive, then $\tilde{M}$ is increasing and since $\|v\| \leq \liminf _{n \rightarrow \infty}\left\|u_{n}\right\|$, we get

$$
\tilde{M}\left(\|v\|^{2}\right) \leq \tilde{M}\left(\left(\liminf _{n \rightarrow \infty}\left\|u_{n}\right\|\right)^{2}\right) .
$$

Because $\tilde{M}$ is continuous and $\left\|u_{n}\right\| \geq 0$ we obtain

$$
\tilde{M}\left(\left(\liminf _{n \rightarrow \infty}\left\|u_{n}\right\|\right)^{2}\right)=\tilde{M}\left(\liminf _{n \rightarrow \infty}\left\|u_{n}\right\|^{2}\right)=\liminf _{n \rightarrow \infty} \tilde{M}\left(\left\|u_{n}\right\|^{2}\right) .
$$

Hence

$$
\tilde{M}\left(\|v\|^{2}\right) \leq \liminf _{n \rightarrow \infty} \tilde{M}\left(\left\|u_{n}\right\|^{2}\right)
$$

Further the functional $u \mapsto(p+1)^{-1} \int_{\Omega}|u|^{p+1} d x+\lambda \int_{\Omega} f(x) u d x$, is weakly continuous then

(8) $\frac{1}{p+1} \int_{\Omega}\left|u_{n}\right|^{p+1} d x+\lambda \int_{\Omega} f(x) u_{n} d x \rightarrow \frac{1}{p+1} \int_{\Omega}|v|^{p+1} d x+\lambda \int_{\Omega} f(x) v d x$.

From (7) and (8) we deduce that $I$ is weakly lower semi continuous and in consequently

$$
\alpha \leq I(v) \leq \liminf _{n \rightarrow \infty} I\left(u_{n}\right)=\alpha,
$$


so $I(v)=\alpha$, which proves that $I$ attains its infinimum at the limit $v$. Lut us prove that $v$ has a negative energy. Denote $\Omega^{+}=\{x \in \Omega ; f(x)>0\}$, $\Omega^{-}=\{x \in \Omega ; f(x) \leq 0\}$ and choose a positive function $\varphi \in H_{0}^{1}(\Omega)$ such that $\operatorname{supp} \varphi \subset \Omega^{+}$. For $t \in(0,1)$ we have

$$
\begin{aligned}
I(t \varphi) & =\frac{1}{2} \tilde{M}\left(\|t \varphi\|^{2}\right)-\frac{1}{p+1} \int_{\Omega}|t \varphi|^{p+1} d x-\lambda \int_{\Omega^{+}} f(x) t \varphi d x-\lambda \int_{\Omega^{-}} f(x) t \varphi d x \\
& \leq \frac{1}{2} M\left(t^{2}\|\varphi\|^{2}\right)\|\varphi\|^{2} t^{2}-\frac{t^{p+1}}{p+1} \int_{\Omega}|\varphi|^{p+1} d x \\
& \leq \frac{1}{2} M\left(\|\varphi\|^{2}\right)\|\varphi\|^{2} t^{2}-\frac{t^{p+1}}{p+1} \int_{\Omega}|\varphi|^{p+1} d x
\end{aligned}
$$

and since $1<p+1<2$, we deduce that $I(t \varphi)<0$ for small values of $t$ and so

$$
I(v)=\inf _{u \in H_{0}^{1}(\Omega)} I(u) \leq \inf _{0<t<1} I(t \varphi)<0 .
$$

$v$ is then a nontrivial global minimum for $I$ with negative energy. Theorem 3 is proved.

Acknowledgement. We are very grateful to the anonymous referee for his valuable comments and suggestions.

\section{References}

[1] Alves, C. O. and Corrêa, F. J. S. A., On existence of solutions for a class of problem involving a nonlinear operator, Comm. Appl. Nonlinear Anal., 8 (2001), 43-56.

[2] Alves, C. O., Corrêa, F. J. S. A. and Ma, T. F., Positive solutions for a quasilinear elliptic equation of Kirchhoff type, Comput. Math. Appl., 49 (2005), 85-93.

[ 3 ] Các, N. P., Gatica, J. A. and Li, Y., Positive solutions to semilinear problems with coefficient that changes sign, Nonlinear Anal., 37 (1999), 501-510.

[4] Corrêa, F. J. S. A. and Menezes, S. D. B., Existence of solutions to nonlocal and singular elliptic problems via Galerkin method, Electron. J. Differential Equations, 2004, No. 19, $10 \mathrm{pp}$.

[5] Dai, Q. and Gu, Y., Positive solutions for non-homogeneous semilinear elliptic equations with data that changes sign, Proc. Roy. Soc. Edinburgh Sect. A, 133 (2003), 297-306.

[6] Kirchhoff, G., Mechanik, Teubner, Leipzig, 1883.

[7] Lions, J. L., On some questions in boundary value problems of mathematical physics, North-Holland Math. Stud., 30, Amsterdam, 1978, pp. 284-346.

[ 8 ] Lions, J. L., Quelques méthodes de résolution des problèmes aux limites non linéaires, Dunod, Gauthier-Villars, Paris, 1969.

[9] Ma, T. F., Remarks on an elliptic equation of Kirchhoff type, Nonlinear Anal., 63 (2005), 1967-1977. 
nuna adreso:

N. Azzouz

Département de Mathématiques

Faculté des Sciences

Université de Tlemcen

BP 119(13000) Tlemcen

Algérie

E-mail: azzouz_n@live.fr
A. Bensedik
Département de Mathématiques
Faculté des Sciences
Université de Tlemcen
BP 119(13000) Tlemcen
Algérie
E-mail: a_bensedik@mail.univ-tlemcen.dz

(Ricevita la 15-an de novembro, 2010)

(Reviziita la 13-an de februaro, 2011) 